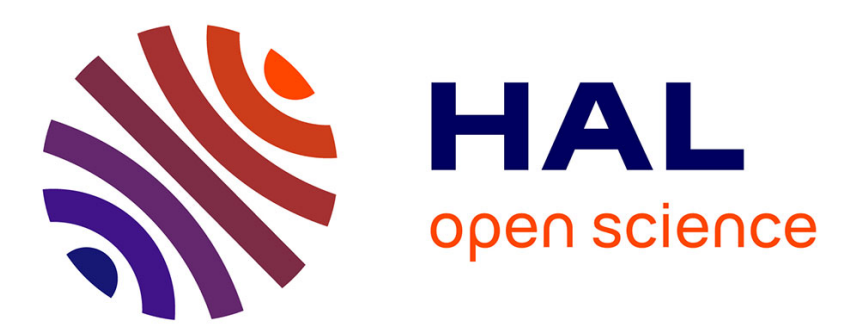

\title{
Strategies of segmental stabilization during gait in Parkinson's disease
}

\author{
Serge Mesure, Jean-Philippe Azulay, Jean Pouget, B. Amblard
}

\section{To cite this version:}

Serge Mesure, Jean-Philippe Azulay, Jean Pouget, B. Amblard. Strategies of segmental stabilization during gait in Parkinson's disease. Experimental Brain Research, 1999, 129 (4), pp.573-581. 10.1007/s002210050927. hal-01644859

\section{HAL Id: hal-01644859 \\ https://hal-amu.archives-ouvertes.fr/hal-01644859}

Submitted on 28 Nov 2017

HAL is a multi-disciplinary open access archive for the deposit and dissemination of scientific research documents, whether they are published or not. The documents may come from teaching and research institutions in France or abroad, or from public or private research centers.
L'archive ouverte pluridisciplinaire HAL, est destinée au dépôt et à la diffusion de documents scientifiques de niveau recherche, publiés ou non, émanant des établissements d'enseignement et de recherche français ou étrangers, des laboratoires publics ou privés. 


\begin{abstract}
This study compared the postural strategies adopted by patients with Parkinson's disease (PD; $n=16)$ during locomotion to those of elderly controls $(n=16)$. We focused mainly on the head and trunk stabilization modes in sagittal and frontal planes. Subjects were asked to walk at their natural speed on an uniformly gray, flat ground. Gait data were recorded before and $1 \mathrm{~h}$ after L-dopa intake and were analyzed by an automatic motion analyser (Elite system). The modes of segmental stabilization adopted by each group were determined by means of the anchoring index, associated with cross-correlation functions between angular movements of pairs of segments. The major findings were: (a) PD patients generally had shorter step length, greater step width, and slower gait velocity than the healthy elderly. (b) No difference in angular dispersion of any anatomical segment studied was observed between the two groups. (c) PD patients had adopted a strategy of head stabilization on the shoulder ("en bloc" functioning of the head-shoulder unit) about the roll axis only. (d) PD patients displayed head and shoulder angular movements around the roll axis that were more correlated than those of controls, confirming their more en bloc functioning. (e) Shoulder and hip were equally stabilized in space in the two groups around the roll axis. (f) There was no difference between the two groups about the pitch axis where an en bloc functioning of the whole trunk was shown. These results are discussed with respect to the similarities observed between the visuo-locomotor PD performances and those of children.
\end{abstract}

S. Mesure $\cdot$ J.P Azulay $\cdot$ J. Pouget

UPRES Physiopathologie du système nerveux,

Hôpital de la Timone, F-13385 Marseille Cedex 05, France

S. Mesure (E) · J.P. Azulay · B. Amblard

UPR Neurobiologie et Mouvements, CNRS,

31 Chemin Joseph Aiguier, BP 71, 13402 Marseille,

Cedex 20, France,

e-mail: mesure@Inf.cnrs-mrs.fr,

Tel.: +33-491-164649, Fax +33-491-775084
Key words Gait analysis · Parkinson's disease .

Segmental stabilization strategies · Visual cues

\section{Introduction}

Walking abilities are almost always impaired in patients with Parkinson's disease (PD). Generally gait disorders are associated with a loss of independence and an increased incidence of falls (Koller et al. 1989; Morris et al. 1994). The gait of PD patients is very stereotyped and clearly recognizable. Gait hypokinesia (slowness) is one of the primary and most frequent movement disorders in PD. During the past 20 years the results of several quantitative studies of walking performances have been published. These mainly demonstrate a decrease in gait velocity associated with a decreased step length, with a relatively maintained cadence (Azulay et al. 1996; Blin et al. 1990; Knutsson 1972; Marsden 1994; Murray et al. 1978). Diener et al. (1990) has reported that electromyographic activities of lower limbs are altered and latencies of muscular responses. A decrease in limb flexion-extension has also been described (Forssberg et al. 1984; Stern et al. 1983). Reduced hip, knee, and ankle rotations in the sagittal plane have been shown to depend on the stage of PD (Murray et al. 1978). In fact, Zijlmans et al. (1996) have distinguished the gait pattern of patients with vascular parkinsonism from that of patients with idiopathic PD, in whom gait velocity was equally reduced. Patients with vascular parkinsonism showed less flexion dystonic posture of the elbow, hip, knee, and trunk throughout the gait cycle than patients with PD. This clearly implies a precise selection of patients with idiopathic PD when analyzing their gait disorders. Until now, although many kinematic data are available on this population, little is known about the angular movements of the upper trunk and the head in walking by PD patients.

An improvement in PD patients' gait by sensory cueing has been demonstrated by several authors. As regards the visual contribution to locomotor control and the effects of visual defects in parkinsonism (Mestre et 
al. 1990), however, they are still only little understood, since visual cues may improve gait velocity as well as induce both festination and freezing (Glickstein and Stein 1991). In fact, transverse visual stripes placed on the floor have been shown to definitely improve gait hypokinesia (Azulay et al. 1999; Martin 1967). The latter authors have reported that this improvement, demonstrated by an increase in step length and mean velocity, results mainly from to the dynamic flow of the retinal image due to the locomotor movements, since the gait pattern remains unchanged when the stripes are viewed under stroboscopic illumination. This improvement in the gait pattern due to visual flow leads to the question of head movements during locomotion.

Human locomotion is a rhythmic activity that induces corresponding rhythmic oscillations of the trunk and the head in both the sagittal and the frontal planes (Grossman et al. 1988). The orientation of the head with respect to space, however, may have to be maintained to serve as an egocentric reference value both for controlling the movement trajectory and for improving the processing of the sensory feedback from the head required to maintain balance (Amblard et al. 1997; Berthoz and Pozzo 1988). In healthy walking adults an efficient stabilization of the head (Berthoz and Pozzo 1988; Grossman et al. 1988) and of the gaze in space (Grossman et al. 1989) have been demonstrated, and it emerges that head orientation along a vertical axis is fairly well stabilized during various locomotor task. Moreover, healthy adults have been shown selectively to adopt a strategy of head stabilization in space in the case of lateral balance difficulty (Assaiante and Amblard 1993). Given the increased visual dependence of PD patients for postural control (Bronstein 1988; Bronstein et al. 1990) and their suspected balance impairment even while walking on flat ground, we could expect that head stabilization in space strategy is a key factor in their locomotor performance.

The present experiment compared the postural strategies of segmental stabilization (those of the head, shoulders, trunk, and pelvis) adopted by PD patients while walking to those of normal elderly subjects in both the sagittal and the frontal planes. We also addressed the question of the possible effect of levodopa on the efficiency of these strategies. PD patients were thus analyzed both before and after treatment with L-dopa.

\section{Materials and methods}

\section{Subjects}

Thirty-two subjects were included in this study: 16 patients with idiopathic PD (9 men, 7 women; mean age $68.8 \pm 4$ years) and the same number of age-matched controls ( 7 men, 9 women; mean age $67.5 \pm 5$ years). All patients were clinically diagnosed as having idiopathic PD according to the United Kingdom Brain Bank diagnostic criteria (Gibb and Lees 1988) and had experienced sustained improvement with dopaminergic treatment: 11 PD patients were in stage II (Hoehn and Yahr) and 5 in stage III. The mean disease duration was 6.3 years. The recordings were carried out at the same hour in the morning. All patients and controls participat- ed with written informed consent, and the protocol was approved by the local ethics committee.

\section{Protocol}

PD patients fasted overnight, without treatment for at least $12 \mathrm{~h}$. They were analyzed in two successive experimental sessions, the first one before (P1) and the other at least $1 \mathrm{~h}$ after treatment (P2). After three consecutive walks in each session to accustom them to the task, subjects were instructed to perform normal locomotion at their natural speed, looking ahead with no specification made about foot positioning. All subjects were walking on an uniformly gray flat ground. The study was performed on a $10-\mathrm{m}$ walkway, and the gait pattern was analyzed during a $3-\mathrm{m}$ walk after the subjects had already walked $4 \mathrm{~m}$.

\section{Data collection}

The kinematics of the body movements were analyzed by means of an optical TV image processor (Elite system) working at $100 \mathrm{~Hz}$ and based on passive markers. The four cameras were placed behind the subjects, at $3.5 \mathrm{~m}$ of the useful acquisition volume $(2.5 \times 3.5 \times 2 \mathrm{~m})$. Under these conditions the system accuracy was such that the error of a single measurement was less than $1.5 \mathrm{~mm}$ on the marker three-dimensional position and about $1^{\circ}$ on the measured angles.

Fifteen retroflective markers ( $6 \mathrm{~mm}$ in diameter) were placed symmetrically in pairs on the subject's back at the following sites: fifth metatarsus joint, external malleolus, tibial plate, posterosuperior iliac crest, acromion, and the base of occipital bone; the three last markers were placed on the sacrum, at the seventh thoracic vertebra, and at the seventh cervical vertebra (Fig. 1). This arrangement of the markers was aimed mainly at analyzing angular movements of the head, shoulder, hip, trunk, and thigh around the anteroposterior and transversal axes.

Data were stored on the harddisk of a PC. Three-dimensional markers coordinates recorded underwent an off-line processing, consisting of tracking, three-dimensional reconstruction and digital filtering for noise reduction by means of a finite impulse response filter (D'Amico and Ferrigno 1990)

\section{Data analysis}

The following parameters were considered in evaluating the subject's performance under the experimental conditions:

- Four gait parameters were calculated in each trial: mean velocity, stride length, cadence, and the gap between the centers of rotation of ankles during double support phases (step width).

- The mean angular and linear head accelerations in the frontal plane in each trial were the averaged values over the whole duration of the trial.

- The angular dispersion $\sigma_{3}$ of any body segment measured was $1 \mathrm{SD}$ of its absolute angular distribution (with respect to external axes) in each trial.

- The anchoring index (AI) was used to compare the stabilization of a given segment with respect both to external space and to the inferior anatomical segment (Amblard et al. 1997; Assaiante and Amblard 1993). AI was given in each trial by the following formula:

\section{$\mathrm{AI}=\left[\left(\sigma_{\mathrm{r}}^{2}\right)-\left(\sigma_{\mathrm{a}}^{2}\right)\right] /\left[\left(\sigma_{\mathrm{r}}^{2}\right)+\left(\sigma^{2}{ }_{\mathrm{a}}\right)\right]$}

where $\sigma_{\mathrm{a}}$ is as previously defined and $\sigma_{\mathrm{r}}$ is the standard deviation of the relative angular distribution (with respect to axes linked to the inferior anatomical segment) of the segment being considered. For example, a positive value of the head AI indicates a better head stabilization in space than on the shoulder (articulated operation of the head-shoulder unit), while a negative value indicates a better head stabilization on the shoulder than in space ("en bloc" functioning of the head-shoulder unit) (Assaiante and Amblard 1993). 
Fig. 1 Experimental set-up (above) and position of the markers. Examples of stick diagrams (below) in the sagittal plane in walking control (left) and PD subject (right). It can be seen that one stride in healthy subject roughly corresponds to two strides in PD patient

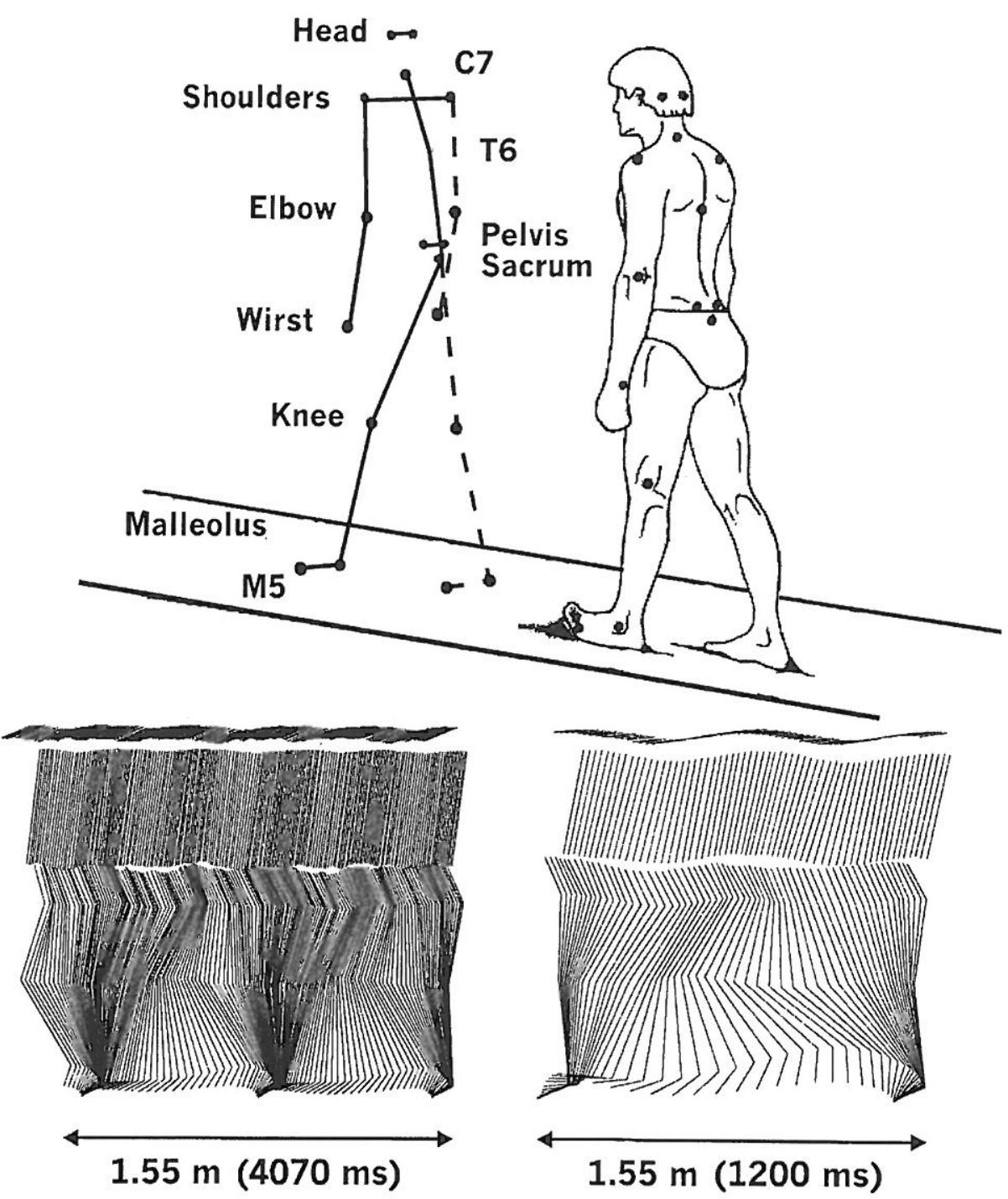

For both $\sigma_{\mathrm{a}}$ and $\mathrm{AI}$ the following anatomical segments considered were: (a) head, shoulder, hip, and thigh in the frontal plane and (b) head and upper and lower trunk in the sagittal plane (see Fig. 1).

- Normalized cross-correlation functions (CCFs) were also calculated between different segmental angular movements about the roll and pitch axes. This was done to evaluate the time lags (abscissa of the cross-correlation peak or trough) between the coordinated movements of two segments and the corresponding correlation coefficient (amplitude of the peak or trough; Amblard et al. 1994). The span of time lags or leads ranged from -600 to $+600 \mathrm{~ms}$ (121 values, 10 -ms intervals). The mean CCFs from three trials in similar conditions was calculated for each subject. Tests of significance of averaged CCF amplitude were performed as previously indicated (Amblard et al. 1997; Mesure et al. 1997).

For each gait parameter linear and angular head acceleration, angular dispersion, $\mathrm{AI}$, and correlation coefficient, three trials were available for averaging under each experimental condition and for each subject. With each angular component (roll and pitch) and treatment condition (before and after) the AI was first used in a one-sample analysis ( $t$ test) against the null hypothesis (Assaiante and Amblard 1993). All the previous parameters were also subjected to analysis of variance to test the significance of compari- sons between experimental conditions or anatomical levels. To test the significance of CCF amplitude, since the successive values of an average CCF were not independent, only 10 equally spaced lags from 121 were used for Student's $t$ test across subjects (Amblard et al. 1994). The probability of false positives in the case of 11 tests was then at most multiplied by 11 (Abdi 1987). A significance level of 0.0046 was thus imposed in each $t$ test to obtain a final significance level of 0.05 . The minimum $P=0.05$ level of significance was adopted throughout data analysis.

\section{Results}

\section{Gait parameters}

During baseline condition the mean gait velocity of PD patients was less than that of controls $(0.76 \pm 0.2 \mathrm{~m} / \mathrm{s}$ vs. $\left.1.13 \pm 0.2 \mathrm{~m} / \mathrm{s} ; F_{1,30}=27.75 ; P<0.001\right)$. The mean stride length was less $(925 \pm 176 \mathrm{~mm}$ vs. $1172 \pm 193 \mathrm{~mm}$; $\left.F_{1,30}=14.48 ; P<0.001\right)$, and the cadence was significantly reduced $\left(99 \pm 11.3\right.$ steps $/ \mathrm{min}$ vs. $116 \pm 10.7 ; F_{1,30}=19.79$; $P<0.001)$. These results were obtained for patients and 
Fig. 2 Mean dispersion in degrees (with standard deviation) of angular movements about the roll (left) and pitch axes (right) in each anatomical segment considered, and each group
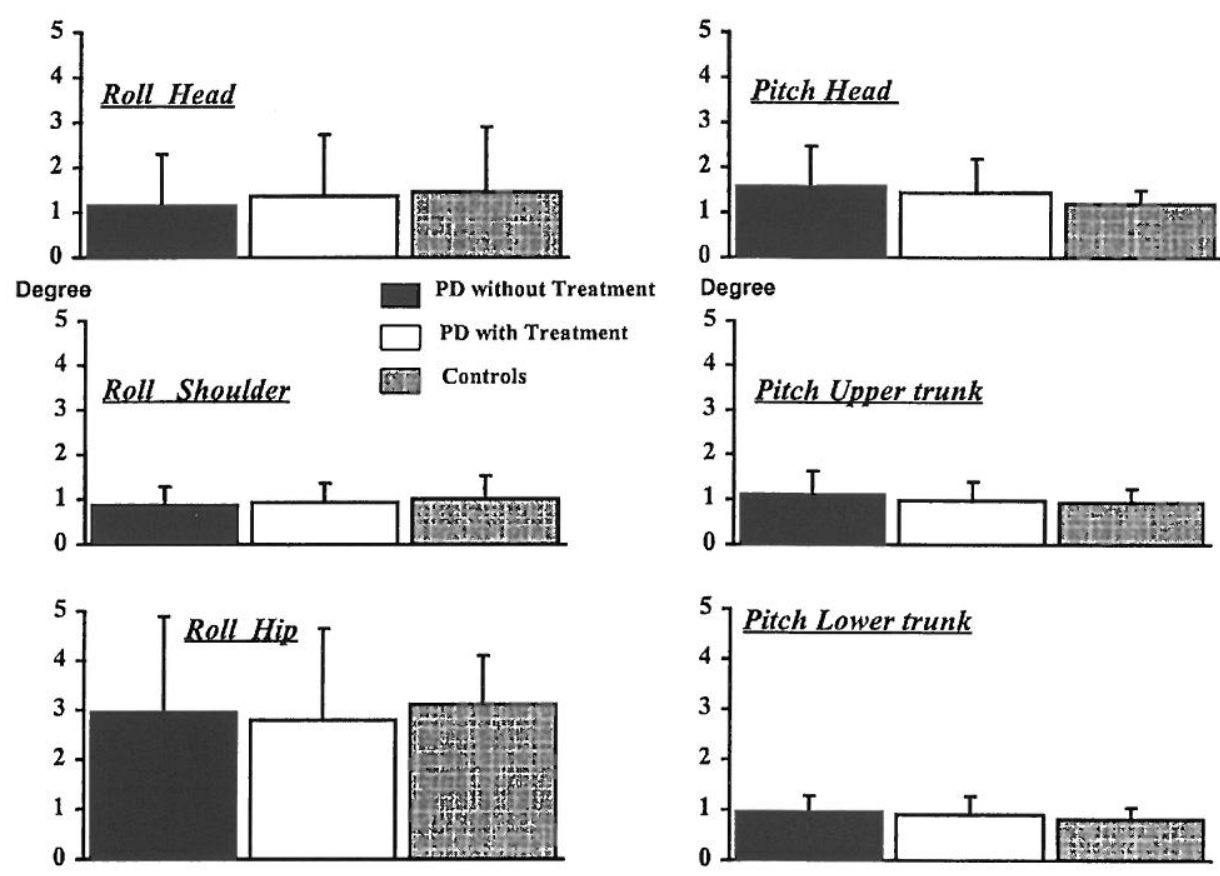

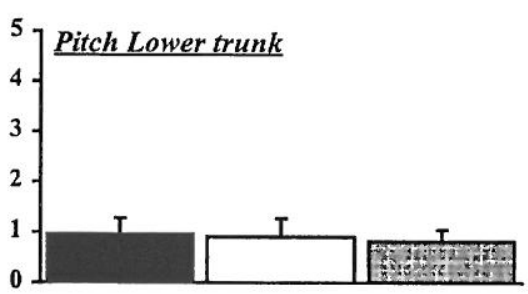

controls walking at their preferred speed, and they showed the same differences commonly reported between these two groups of subjects in the literature.

The last parameter analyzed here was the step width for which until now there has been no clear information on PD patients. The step width was greater both before $\left(121.1 \pm 67.5 \mathrm{~mm} ; F_{1,28}=5.21 ; P<0.05\right)$ and after treatment in PD patients $(118.7 \pm 66 \mathrm{~mm})$ than in controls $\left(88.8 \pm 35.7 \mathrm{~mm} ; F_{1,29}=4.538 ; P<0.05\right)$. L-Dopa treatment had no effect on step width. Step width thus appeared to allow us to distinguish between PD patients and controls, whereas it was independent of L-dopa treatment.

\section{Linear and angular head accelerations}

The mean angular acceleration of the head around the roll axis while walking was significantly higher in healthy subjects $\left(50.3 \pm 15.7^{\circ} / \mathrm{s}^{2}\right)$ than in in $\mathrm{PD}$ patients both before $\left(34.4 \pm 10 \% \mathrm{~s}^{2}, t=5.74, P<0.00000015\right)$ and after $\left(36.4 \pm 11.6^{\circ} / \mathrm{s}^{2}, t=4.86, P<0.000005\right)$ L-dopa intake. LDopa treatment had no effect on these head accelerations.

The mean linear acceleration of the head along the vertical axis was significantly greater in healthy subjects $\left(63.4 \pm 44.7 \mathrm{~m} / \mathrm{s}^{2}\right)$ than in PD patients both before $\left(31.7 \pm 25.8 \mathrm{~m} / \mathrm{s}^{2}, t=3.58, P<0.0008\right)$ and after $\left(27.5 \pm 22.2 \mathrm{~m} / \mathrm{s}^{2}, t=5.55, P<0.000002\right) \mathrm{L}$-dopa intake. The mean linear acceleration of the head along the lateral axis was significantly higher in healthy subjects $\left(60.9 \pm 33.4 \mathrm{~m} / \mathrm{s}^{2}\right)$ than in PD patients both and before $\left(40.3 \pm 29.2 \mathrm{~m} / \mathrm{s}^{2}, \mathrm{t}=2.84, P<0.007\right)$ and after $(46.6 \pm 34.7$ $\left.\mathrm{m} / \mathrm{s}^{2}, t=-2.13, P<0.04\right)$ L-dopa intake. L-Dopa treatment had no effect on the head linear accelerations along either the vertical or the horizontal axes.
Segmental angular dispersions

The averaged head, shoulder, and hip dispersions around the roll axis are shown in Fig. 2 (left), as a function of the experimental conditions. The head and shoulder roll dispersions ranged roughly between $1^{\circ}$ and $1.5^{\circ}$, which provides an initial idea of the degree of head and shoulder stability. The hip roll dispersions was roughly $3-3.5^{\circ}$ and were significantly higher than those of the head and shoulder $\left(F_{2,88}=20.26, P<0.001\right)$, clearly indicating some decline from the hip to the head of the lateral oscillations of the body induced downwards by the locomotor movement.

Few differences between healthy elderly and PD groups were observed. At the hip level, larger standard deviations were noted in the PD patients than in controls $\left(F_{1,14}=8.63, P<0.01\right)$. The head dispersions were similar in the two groups, indicating a similar head stability while walking.

The mean head, upper, and lower trunk dispersions about the pitch axis are shown in Fig. 2 (right). These ranged roughly between $1^{\circ}$ and $2^{\circ}$, with no significant difference between anatomical levels or experimental conditions. This is a first indication that anterior-posterior angular movements produced by locomotor displacements are roughly comparable in PD patients and the normal population.

\section{Anchoring index}

The head, shoulder, and hip AI around the roll axis are shown in Fig. 3 (left) as a function of the experimental 
Fig. 3 Head, shoulder and hip $\mathrm{AI}$ (with standard deviation) about the roll (left) and pitch (right) in each anatomical segment and each group
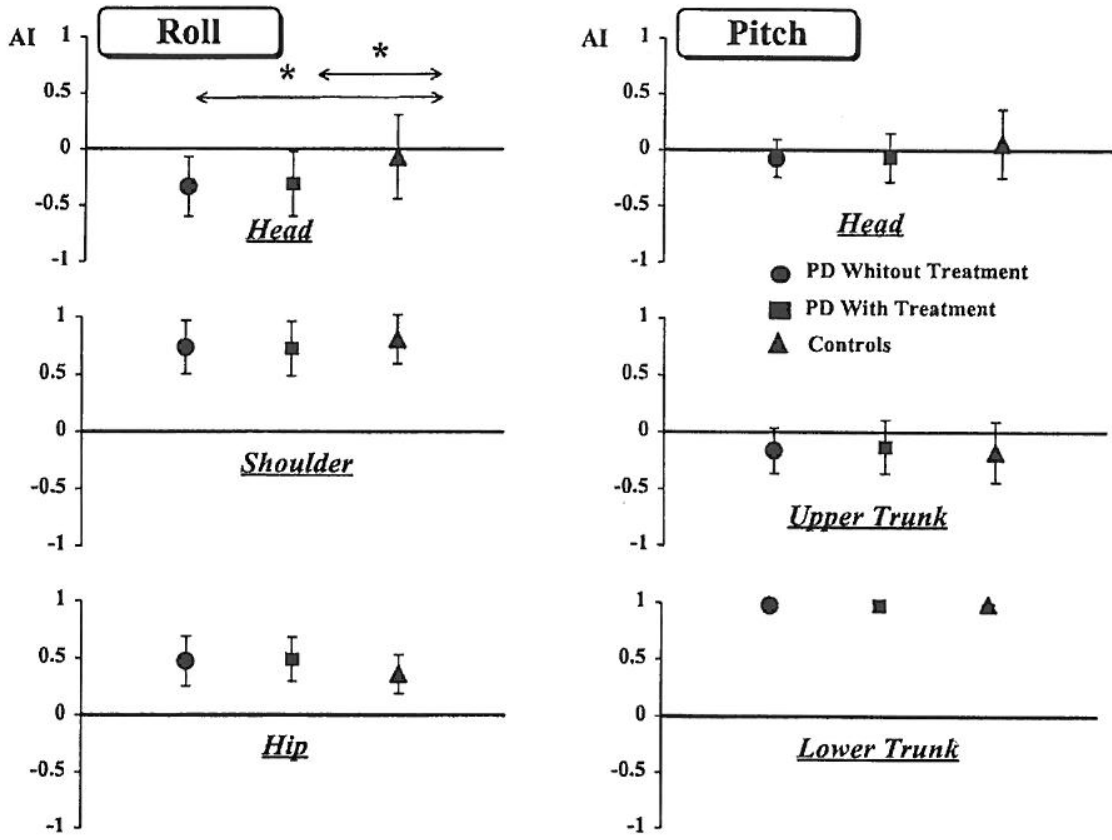

conditions. The corresponding Student's $t$ analysis is given in Table 1. In the control group the head AI did not significantly from zero, indicating no preferred head stabilization either in space or on the shoulder. In the PD group, by contrast, the head AI was always significantly negative, indicating an en bloc functioning of the headshoulder unit in the frontal plane. This strategy was not affected by L-dopa treatment. A significant difference between normals and the PD group was found both with $\left(F_{1,29}=6.80, P<0.05\right)$ and without $\left(F_{1,29}=6,75, P<0.05\right)$ treatment.

The shoulder and hip AI were always significantly positive in both groups and under all experimental conditions $(P<0.001)$. This indicates efficient shoulder and hip stabilization in space strategies. We found no difference between controls and PD subjects. The latter had thus adopted the same shoulder and hip stabilization strategies while walking as had controls, and these strategies were not affected by the treatment conditions.

The head, upper, and lower trunk AI values around the pitch axis are shown in Fig. 3 (right). The corresponding Student's $t$ analysis is given in Table 1 . The head AI was never significantly different from zero regardless of the group and the treatment condition. This indicates that no preferred head strategy around the pitch axis was adopted either in space or on the shoulder, in the controls or in PD patients. Moreover, there was no difference between groups and/or experimental conditions.

In both PD patients and the control group the upper trunk AI was always significantly negative, both with and without treatment. This indicates an en bloc functioning of the whole trunk around the pitch axis while walking. There was no difference between groups or experimental conditions. The lower trunk AI was always highly positive (near +1$)$ in all groups and experimental conditions.
Table 1 One sample analysis (Student's $t$ value with significance level) of the roll and pitch AI in each anatomical segment considered and each group

\begin{tabular}{llll}
\hline Normal Ground & $\begin{array}{l}\text { Mean } \\
(\mathrm{ms})\end{array}$ & $t$ & $P$ \\
\hline Head roll & & & \\
Controls & -0.068 & NS & - \\
PD with treatment & -0.334 & -4.828 & 0.0002 \\
PD without treatment & -0.302 & -4.080 & 0.0009 \\
Shoulder roll & & & \\
Controls & 0.808 & 14.12 & 0.0000000011 \\
PD with treatment & 0.737 & 12.18 & 0.0000000035 \\
PD without treatment & 0.725 & 11.64 & 0.0000000065 \\
Hip roll & & & \\
Controls & 0.378 & 8.52 & 0.00000064 \\
PD with treatment & 0.472 & 8.34 & 0.00000052 \\
PD without treatment & 0.488 & 9.80 & 0.000000098 \\
Head pitch & & & \\
Controls & 0.061 & NS & - \\
PD with treatment & -0.74 & NS & - \\
PD without treatment & -0.064 & NS & - \\
Upper trunk pitch & & & \\
Controls & -0.174 & -2.435 & 0.028 \\
PD with treatment & -0.162 & -3.009 & 0.08 \\
PD without treatment & -0.127 & -2.111 & 0.052 \\
Lower trunk pitch & & & \\
Controls & 0.988 & 564.38 & 0.000 \\
PD with treatment & 0.977 & 294.49 & 0.000 \\
PD without treatment & 0.090 & 305.16 & 0.000 \\
\hline & & & \\
\hline
\end{tabular}

This simply indicates that the anterior-posterior oscillations of the thighs during locomotion always displayed much higher amplitudes than those of the lower trunk. PD patients thus displayed an en bloc functioning of the head-shoulder unit around the roll axis, and a similar 


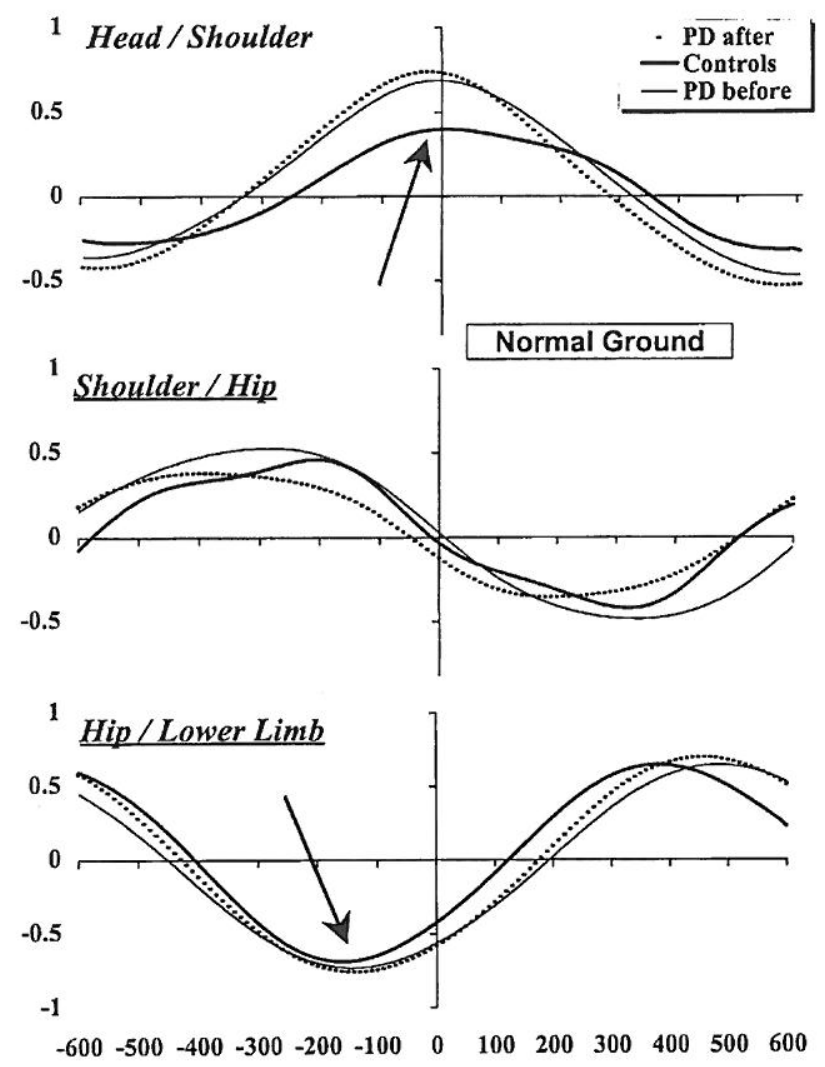

(ms)

Fig. 4 Examples of mean CCFs between head and shoulder, shoulder and hip, and hip and lower limb angular movements about the roll axis while walking on normal ground, in each group. Arrows CCF peak (or trough) whose abscissa was the nearest to zero (see Table 2). Negative abscissa of the head-shoulder CCF peak means that head lags the shoulder, the two moving in the same direction. Similarly, negative abscissa of the hip-lower limb CCF trough means that hip lags the lower limb, the two moving in opposite directions

strategy was applied to the whole trunk in the sagittal plane. This confirms that these patients were clearly accustomed to minimizing the number of degrees of freedom to be controlled simultaneously during movement.

The main result to emerge from this experiment was thus that the head was found to be equally stabilized around the roll axis during locomotion in PD patients and in controls, although by means of different head stabilization modes: PD patients minimized their head movements by stabilizing this segment on shoulder and reducing gait velocity, whereas controls did not adopt a preferred head mode of stabilization either in space or on shoulder despite their greater locomotor speed.

Time and space relationships between segmental movements

Examples of the CCFs are shown in Fig. 4. Since the main differences in the strategies of segmental stabiliza-
Table 2 Correlation coefficients $(r)$ with statistical analysis (Student's $t$ value with significance level) and latencies between angular movements of head and shoulder, and hip and lower limb about the roll axis, in each group

\begin{tabular}{lrrrl}
\hline Normal Ground & $\begin{array}{r}\text { Peak } \\
(\mathrm{ms})\end{array}$ & $\begin{array}{l}\text { Mean } \\
(\mathrm{ms})\end{array}$ & \multicolumn{1}{l}{$t$} & $P$ \\
\hline Controls & 0 & 0.40 & 4.5891 & 0.0011 \\
PD without treatment & -10 & 0.69 & 4.8270 & 0.0008 \\
PD with treatment & -20 & 0.74 & 4.8891 & 0.0007 \\
Controls & -150 & -0.69 & -4.8620 & 0.0008 \\
PD without treatment & -140 & -0.73 & -4.4369 & 0.0013 \\
PD with treatment & -140 & -0.76 & -4.5432 & 0.0012
\end{tabular}

Positive $r$ values indicate angular movements performed in the same direction; negative latencies indicate head (or hip) following shoulder (or lower limb) lateral movements

tion between PD and control groups were found around the roll axis, we consider only the CCFs between segmental movements in the lateral plane. In this plane the patterns of CCFs were found to depend more on the pair of anatomical levels considered than on the experimental condition. Due to the same cadence in their locomotor patterns CCFs are fairly similar in all groups of subjects.

The correlation coefficient and the latency between coordinated movements were, respectively, the amplitude and the abscissa of the peaks or trough of the CCF, whose abscissa was the nearest to zero (Amblard et al. 1994). For example, the negative latency of the headshoulder CCF peak (Fig. 4) means that the head movement followed those of the shoulder. The positive correlation coefficient indicates that the head and shoulder moved in the same direction. Table 2 presents the correlation coefficients (with statistical significance; see above) and the latencies between coordinated oscillations about the roll axis of the head and shoulder on the one hand, and on the other hand of the hip and lower limb.

In the case of head-shoulder coordination there was always a slight or no delay between movements of the two segments (head following shoulder) performed in the same direction (in phase oscillatory movements as indicated by the positive correlation coefficient). This mode of coordinated and almost simultaneous movement did not depend on the experimental conditions. The correlation coefficient was lower in controls than in PD patients ( $t=2.187, P<0.05$, before treatment; $t=2.383$, $P<0.05$ after treatment). This is in agreement with the en bloc functioning of the head-shoulder unit in PD patients in all the experimental conditions considered. Moreover, there was no difference before and after treatment in PD patients.

The shoulder-hip CCFs indicated roughly a $90^{\circ}$ phase shift between angular movements of the two segments, and it was therefore impossible to distinguish between phase lag and lead between one and the other segment. Moreover, there was no statistically significant difference between groups. 
The negative hip-thigh CCF peak around $-150 \mathrm{~ms}$ corresponded to coordinated movements in opposite directions, with thigh in advance of hip movements. In PD patients the treatment induced a slight decrease in the correlation coefficient while walking on the ground $(t=2.406, P<0.05)$. This indicates than PD patients were closer to controls after than before treatment. There was, however, no corresponding difference in the hip AI.

\section{Discussion}

In this study we found that most of the spatiotemporal parameters of PD patients differ from those obtained in normal aged-matched controls, as has been noted in previous studies quantifying gait abnormalities in PD patients (Blin et al. 1991; Knutsson 1972; Morris et al. 1994; Murray et al. 1978; Pedersen et al. 1991; Stern et al. 1983). Typically we found that both before and after L-dopa treatment PD patients display shorter step length, slower free-walking speed, and walk with their feet more apart than controls in the lateral plane.

The presence of an abnormally large step width in PD patients has not been described in previous studies on PD patients' gait. Zijlmans et al. (1996), who measured step width by making footprints with magnesium powder while wearing standard shoes, found differences in the step width between healthy subjects and patients with vascular PD $(50 \pm 30$ and $90 \pm 40 \mathrm{~mm}$, respectively). Nutt et al. (1993) noted variability only in stride width in patients with advanced PD. Vieregge et al. (1997), however, found no significant differences between PD patients and age-matched healthy subjects in "step width and its coefficient of variation (the latter related to postural imbalance in locomotion)." Our results thus rather confirm those of Zijlmans and colleagues. The discrepancies between previous studies could be partly due to the methods of measurements and/or the Hoehn and Yahr stages of the patients studied. Previous studies did not make use of such modern technical equipment such the Elite system, which provides reproducible and precise kinematic data and demonstrates a slight but significant increase in the step width of PD patients. Our results are in accord with the idea of balance difficulties encountered by PD patients. Widening the stance base has been found to reduce lateral body sway and to a lesser degree, anteroposterior motion (Day et al. 1990). Similarly, if balance is perceived as less secure by PD patients, their gait pattern is altered accordingly (Nutt et al. 1993), namely by increasing step width. We have also shown that L-dopa treatment has no effect on step width. This is coherent with the fact that L-dopa treatment does not improve balance in PD patients. Step width thus illustrates a locomotor adaptation of this population and can be considered as an invariant of their locomotor pattern (Borghese et al 1996), as with cadence and step length.

Our second purpose was to compare the strategies of segmental stabilization adopted by PD patients to those of the normal elderly. The hypothesis was that head and trunk angular oscillations occurring while walking indicate the dynamic balance performance. The main result to emerge from this study was that the PD patients adopt a head stabilization on the shoulder strategy. This was demonstrated both by the negative AI values and the increase in the positive head-shoulder correlation coefficient compared to controls. The increase in the correlation coefficient of could correspond to a stiffening of the neck muscles. An initial interpretation of this en bloc functioning of the head-shoulder unit is that it is adopted in response to locomotor balance difficulty in the frontal plane encountered by PD patients. Although we did not quantitate balance difficulties in $\mathrm{PD}$, impaired postural control was shown in five of our patients, who were in Hoehn and Yahr stage III. This interpretation must be confirmed by further investigations. An alternative interpretation of the en bloc functioning of the head trunk unit in the lateral plane is that the tone control is abnormal in PD patients. The fixation of the head on the shoulder could not have been secondary to locomotor problems but merely a consequence of basal parkinsonian dystonic abnormalities of posture and tone. We cannot absolutely exclude this interpretation. However, several findings make this unlikely. First, the head was stabilized only around the roll axis, whereas abnormalities of tone in PD are known to involve mainly the muscles which cause flexion extension of the head in the sagittal plane. A second argument is that the en bloc strategy concerned only the head and shoulders and not other anatomical levels. Lastly, if the tone abnormalities did play a major role in the en bloc strategy, levodopa intake should have modified this strategy, and we did not find such effect. The en bloc functioning of the head-trunk unit was largely resistant to L-dopa treatment. We would have expected in PD patients a similar blocking of the whole trunk as we observed in the sagittal but not in the frontal plane, despite the classically known rigidity of PD patients. Interestingly, PD patients did not show more trunk rigidity than controls, since both their shoulder AI and the amplitudes of angular movements at shoulder and lower trunk levels never differed from those of healthy aging subjects. Finally, as with the controls, they were also able to stabilize their hips in space while walking. PD patients were thus found to adopt roughly the same movement strategies as controls at the trunk level, although with lower walking speed and shorter step length. The head was thus the only segment whose stabilization strategy was affected by PD.

The strategy adopted by PD patients, consisting of blocking the head on the trunk to minimize the number of degrees of freedom to be controlled simultaneously during movement (Bernstein 1967) can be considered as a simplifying strategy, concerning selectively the plane perpendicular to that of the displacement. A similar strategy has been described in children and adults under difficult locomotor conditions (Assaiante and Amblard 1993, Assaiante et al. 1998; Pozzo et al. 1995). Forssberg (1985) and Forssberg et al. (1984) have also described close similarities between the gait pattern of 
the PD patients and that of children who have not yet developed a plantigrade gait ( $<18-24$ month). These authors interpreted their observations in PD patients as regression to an immature gait. According to them, it is likely that the primitive gait in small children is transformed to the adult type by a decreasing effect from the brain, and that this effect is lost in PD due to a lack of dopaminergic transmission in basal ganglia. In our study, however, the lateral head-trunk blocking was not affected by medication. We have also shown that PD patients walk with their feet farther apart laterally than controls. The same strategy, aimed at facilitating balance control while walking, has also been described in children. Infants cannot reduce the gap between their feet while walking before about 2 years of walking experience (Brenière et al. 1989; Bril and Brenière 1988). The en bloc functioning of the head-shoulder unit in walking PD patients can also be regarded as regression to an immature postural strategy similar to that previously described in children up to 6 years of age by Assaiante et Amblard (1993). In young children, however, the en bloc functioning of the head-shoulder unit is generalized to all the degrees of freedom of the neck, whereas in PD patients it concerns only the roll angle. Moreover, visual dependency of $\mathrm{PD}$ patients previously described in the control of locomotion (Azulay et al. 1999) resembles that of infants and children, in whom vision gradually acquires a predominant role during various transition phases in the course of their early sensory-motor development (Butterworth 1986; Forssberg 1985; Shumway-Cook and Woollacott 1985; Woollacott et al. 1987). The transition to a pathological state such as PD thus appears to induce an increased visual dependence similar to that of children.

Given the visual dependence assumed in PD patients in various motor tasks such as control of posture (Bronstein et al. 1990) and locomotion (Azulay et al. 1999), it may be surprising that they do not stabilize their heads in space while walking. A "head stabilization in space strategy" would have improved the visual processing needed for dynamic balance to be maintained in PD patients. If their heads follow their trunk movements during locomotion, vision of the environment can be blurred and therefore impair the visual contribution to locomotor balance. It should be noted, however, that PD patients have a decreased locomotor velocity, resulting in lateral head and trunk oscillations of similar amplitudes than those of age-matched controls. This suggests that PD patients do not experience more visual blurring while walking than healthy subjects. Moreover, the vestibulo-ocular reflex (VOR), which stabilizes the gaze in space and stabilizes the retinal image (Grossman et al. 1989), has not been reported to be markedly affected in PD. According to Rottach et al. (1996), the horizontal VOR, with or without visual enhancement, is unaffected in idiopathic PD.

In advanced PD disease only, White et al. (1983) have described subnormal VOR gains in darkness during sinusoidal whole-body rotation at frequencies from 0.3 to
$3.0 \mathrm{~Hz}$, this frequency domain including that of locomotion. The same authors also show that the gains of patients with advanced disease are significantly lower than those of controls when fixating on a stationary target. Our patients could thus be considered as having almost normal VOR since most of them were in Hoehn and Yahr stage II. We have shown that their mean head linear and angular accelerations in the frontal plane while walking are in the order of $40 \mathrm{~m} / \mathrm{s}^{2}$ and $35^{\circ} / \mathrm{s}^{2}$, respectively, which are values substantially higher than the thresholds of both otoliths (approximately $0.05 \mathrm{~m} / \mathrm{s}^{2}$ ) and semicircular canals (approximately $0.2 \% \mathrm{~s}^{2}$ ), according to Henn et al. (1980). Moreover, these mean head linear and angular accelerations are lower than in age-matched healthy subjects $\left(60 \mathrm{~m} / \mathrm{s}^{2}\right.$ and $50 \% / \mathrm{s}^{2}$, respectively) Therefore vision of the environment while walking is presumably not more blurred in our patients than in controls, despite the en bloc functioning of the head-trunk units of the first and the limited VOR in the frontal plane. Blocking the head on the shoulder has the advantage of providing the subject with direct information on lateral body balance, thus facilitating the use of both visual cues and extraocular proprioception as error signals for postural control.

Finally, the good synchronism between coordinated head and shoulder movements in PD patients is coherent, with some locking of the neck joint rather than with anticipation of upward perturbations due to locomotor trunk movements. This en bloc functioning may be interpreted as a freezing strategy of the degrees of freedom to be controlled simultaneously and may explain the PD patients' inability to respond correctly to unpredictable external stimuli.

Regardless of the strategy adopted, it can be concluded that the control of locomotor balance entails limiting the head angular oscillations, especially in the lateral plane. PD patients achieve this mainly by a simplified strategy consisting in blocking the head on the trunk. This strategy is probably chosen principally because of PD patients' limited sensory-motor abilities. Vision, which these patients use predominantly for compensation, thus benefits from the limitation of head movements and efficiently ensures its contribution to lateral balance control by reducing lateral trunk oscillations.

Acknowledgement We are grateful to Dr. J. Massion for critically reviewing the manuscript. This study was supported by a grant from the French Ministry of Health (Programme Hospitalier de Recherche Clinique 1996).

\section{References}

Abdi $H$ (1987) Introduction au traitement statistique des données expérimentales. Presses Universitaires de Grenoble, Grenoble

Amblard B, Assaiante C, Lekhel H, Marchand AR (1994) Statistical approach to sensorimotor strategies: conjugate cross-correlations. J Mot Behav 26:103-112

Amblard B, Assaiante C, Fabre JC, Mouchnino L, Massion J (1997) Voluntary head stabilization in space during oscillatory trunk movements in the frontal plane performed in weightlessness. Exp Brain Res 114:214-225 
Assaiante C, Amblard B (1993) Ontogenesis of head stabilization in space during locomotion in children - influence of visual cues. Exp Brain Res 93:499-515

Assaiante C, Thomachot B, Aurenty R, Amblard B (1998) Organization of lateral balance control in toddlers during the first year of autonomous walking. J Mot Behav 30:114-129

Azulay JP, Vanden Brand I, Mestre D, Blin O, Sangla I, Pouget J, Serratrice G (1996) Analyse cinematique de la marche du parkinsonien: effet de la levodopa et de stimulations visuelles. Rev Neurol (Paris) 152:128-134

Azulay JP, Mesure S, Amblard B, Blin O, Sangla I, Pouget J (1999) Visual control of locomotion in Parkinson's disease. Brain 122:111-120

Bernstein N (1967) The coordination and regulation of movements. Pergamon, Oxford

Berthoz A (1991) Les systémes sensorimoteurs. Mécanismes et propriétés adaptatives. CNES report. Séminaire de Prospective Microgravité, pp 22-27

Berthoz B, Pozzo T (1988) Intermittent head stabilization during postural and locomotory tasks in humans. In: Amblard B, Berthoz A, Clarac F (eds.) Posture and gait: development, adaptation and modulation. Elsevier, Amsterdam, pp189-198

Blin O, Ferrandez A, Serratrice G (1990) Quantitative analysis of gait in Parkinson patients: increased variability of stride length. J Neurol Sci 98:91-97

Blin O, Ferrandez A, Pailhous J, Serratrice G (1991) Dopa-sensitive and dopa-resistant gait parameters in Parkinson's disease. J Neurol Sci 103:51-54

Borghese NA, Bianchi L, Lacquaniti F (1996) Kinematic determinants of human locomotion. J Physiol (Lond) 494:863-879

Brenière Y, Bril B, Fontaine R (1989) Analysis of the transition from upright stance to steady state locomotion in children with under 200 days of autonomous walking. J Mot Behav 21: 20-37

Bril B, Brenière Y (1988) Do temporal invariances exist as early as the first six months of independent walking?. In: Amblard B, Berthoz A, Clarac F (eds) Posture and gait: development, adaptation and modulation. Elsevier, Amsterdam, pp 23--31

Bronstein A (1988) Evidence for a vestibular input contributing to dynamic head stabilization in man. Acta Otolaryngol (Stockh) $105: 1 \ldots 6$

Bronstein A, Hood J, Gresty M, Panagi C (1990) Visual control of balance in cerebellar and parkinsonian syndromes. Brain 113: 767-779

Butterworth G (1986) Motor development in children: aspects of coordination and control. In: Wade $\mathrm{M}$, Whiting $\mathrm{H}$ (eds) Themes in motor development. Nijhoff, Dordrecht

D'Amico M, Ferrigno G (1990) Technique for the evaluation of derivates from noisy biomechanical displacement data using a model-based bandwidth-selection procedure. Med Biol Eng Comput 28:407-415

Day B, Steiger M, Thompson M, Marsden C (1990) Effect of stance width on body movement when standing. In Brandt $\mathrm{T}$, Paulus W, Bles W (eds) Disorders of posture and gait. Thieme, Stuttgart, pp 37-40

Diener H, Dichgans J, Guschlbauer B, Bacher M, Rapp H, Langenbach P (1990) Associated postural adjustments with body movement in normal subjects and patients with parkinsonism and cerebellar disease. Rev Neurol (Paris) 146: $555-563$

Forssberg H (1985) Ontogeny of human locomotor control. I. Infant stepping, supported locomotion and transition to independent locomotion. Exp Brain Res 57:480-493

Forssberg H, Johnels B, Steg G (1984) Is parkinsonian gait caused by regression to an immature walking pattern? Adv Neurol 40:375--379

Gibb W, Lees A (1988) The relevance of the lewy body to the pathogenesis of idiopathic Parkinson's disease. J Neurol Neurosurgery Psychiatry 51:745-752
Glickstein M, Stein J (1991) Paradoxical movement in Parkinson's disease. Trends Neurosci 14:480-482

Grossman GE, Leigh RJ, Abel LA, Lanska DJ, Thurston SE (1988) Frequency and velocity of rotational head perturbations during locomotion. Exp Brain Res 70:470-476

Grossman GE, Leigh RJ, Bruce EN, Huebner WP, Lanska DJ (1989) Performance of the human vestibuloocular reflex during locomotion. J Neurophysiol 62:264-272

Henn V, Cohen B, Young LR (1980) Visual-vestibular interaction in motion perception and the generation of nystagmus. Neurosci Res Program Bull 18:459-651

Keshner E, Cromwell R, Peterson B (1992) Head stabilization during vertical seated rotations and gait. In Woollacott $M$ Horak F (eds) Posture and gait: control mechanisms. University of Oregon Press, Portland, pp 105-108

Knutsson E (1972) An analysis of parkinsonian gait. Brain 95: 475-486

Koller W, Glatt S, Vetere B, Hassanein R (1989) Falls and Parkinson's disease. Clin Neuropharmacol 12:98--105

Marsden C (1994) Parkinson's disease. J Neurol Neurosurg Psychiatry $57: 672-681$

Martin P (1967) The basal ganglia and posture. Pitman, London

Mestre D, Blin O, Serratrice G, Pailhous J (1990) Spatio-temporal contrast sensitivity differs in normal aging and parkinson's disease. Neurology 40:1710-1714

Mesure S, Amblard B, Crémieux J (1997) Effect of physical training on head-hip coordinated movement. Neuroreport 8:35073512

Morris M, Iansek R, Matyas T, Summers J (1994) Ability to modulate walking cadence remains intact in Parkinson's disease. $J$ Neurol Neurosurg Psychiatry 57:1532-1534

Murray M, Sepic B, Gardner M, Downs W (1978) Walking patterns of men with parkinsonism. Am J Phys Med Rehabil $57: 278-294$

Nutt JG, Marsden C, Thompson P (1993) Human walking and higher-level gait disorders, particularly in the elderly. Neurology $43: 268-279$

Pedersen S, Eriksson S, Oberg B (1991) Effects of withdrawal of antiparkinson medication on gait and clinical score in the Parkinson patient. Acta Neurol Scand 84:7-13

Pozzo T, Levik Y, Berthoz A (1995) Head and trunk movements in the frontal plane during complex dynamic equilibrium tasks in humans. Exp Brain Res 106:327-338

Rottach KG, Riley DE, DiScenna AO, Zivotofsky AZ, Leigh RJ (1996) Dynamic properties of horizontal and vertical eye movements in parkinsonian syndromes. Ann Neurol 39:368377

Shumway-Cook A, Woollacott M (1985) The growth of stability: postural control from a developmental perspective. J Mot Behav 17:131-147

Stern G, Franklyn S, Imms F, Prestidge S (1983) Quantitative assessments of gait and mobility in Parkinson's disease. J Neural Transm 19:201-214

Vieregge P, Stolze H, Klein C, Heberlein I (1997) Gait quantification in Parkinson's disease - locomotor disability and correlation to clinical rating scales. J Neural Transm 104:237-248

White OB, Saint-Cyr JA, Sharpe JA (1983) Ocular motor deficits in Parkinson's disease. The horizontal vestibulo-ocular reflex and its regulation. Brain 106:555-570

Woollacott M, Debu B, Mowatt M (1987) Neuromuscular control of posture in the infant and child: is vision dominant? J Mot Behav 19:167-186

Zijlmans J, Poels P, Duysens J, Van der Straaten J, Thien T, VanHof M, Thijssen H, Horstink M (1996) Quantitative gait analysis in patients with vascular Parkinsonism. Mov Disord 11:501-508 